\title{
Network Developments and Monitoring in Internet2
}

\author{
Eric Boyd ${ }^{1}$ \\ Internet2 \\ 1000 Oakbrook Drive, Suite 300, Ann Arbor, Michigan, USA \\ E-mail: eboyd@internet2.edu
}

\section{Susan Evett}

Internet2

1000 Oakbrook Drive, Suite 300, Ann Arbor, Michigan, USA

E-mail: sevett@internet2.edu

Given that performance is excellent across backbone networks, and that performance is a problem end-to-end, it is clear that problems are concentrated towards the edge and in network transitions. To achieve good end-to-end performance, we need to diagnose (understand the limits of performance) and address (work with members and application communities to address those performance issues). We envision readily available performance information that is easy to find, ubiquitous, reliable, valuable, actionable (analysis suggests course of action), and automated (applications act on data received).

The Internet2 End-to-End Performance Initative (E2Epi) currently focuses on the development and widespread deployment of perfSONAR [1][2], an international consortium developing a performance middleware architecture and a set of protocol standards for inter-operability between measurement and monitoring systems. perfSONAR is a set of open source web services that can be added, piecemeal, and extended to create a performance monitoring framework. It is designed to be standards-based, modular, decentralized, and open source. This makes it applicable to multiple generations of network monitoring systems and encourages outside development while still allowing it to be customized for individual science applications. perfSONAR is a joint effort of ESnet, GÉANT2 JRA1, Internet2, and RNP.

The Internet2 Network is a hybrid optical and IP network, that offers dynamic and static wavelength services. The Internet2 Network Observatory supports three types of services: measurement, co-location, and experimental servers to support specific projects. The Observatory collects data and makes it publicly available.

Lighting the Blue Touchpaper for UK e-Science - Closing Conference of ESLEA Project The George Hotel, Edinburgh, UK 26-28 March, 2007

\footnotetext{
$1 \quad$ Speaker
} 


\section{Internet2 Network}

The Internet2 Network is a hybrid optical and IP network, that offers dynamic and static wavelength services. The fiber and equipment on which the Internet2 Network runs is dedicated to Internet2's use; Level 3 provides the network and service level maintenance. The platform supports production services (IP, static waves, and dynamic waves) as well as experimental projects, such as HOPI (Hybrid Optical/Packet Infrastructure).

The deployment of the new Internet2 Network will be complete by July 15; 2007. In March, Internet2 began exploring a merger with National Lambda Rail (NLR) with a goal of consolidating national higher education and research (R\&E) networking organizations. A technical team began considering what the merged technical infrastructure would look like. A decision on this potential collaboration is expected by June 2007.

\section{Performance Middleware}

Science is a global community; networks link scientists and collaborative research occurs across network boundaries. For the scientist, the value of the network is the achieved network performance. Scientists should not have to focus on the network; good end-to-end performance should be a "given." For example, the Large Hadron Collider is an international physics facility located at CERN in Switzerland. This effort involves major U.S. involvement, with 2 major U.S. data repositories (PetaBytes/year), 17 U.S. institutions providing data analysis and storage, and 68 universities and National Laboratories with scientists looking at the data. There are dedicated transatlantic networks connecting the U.S. to CERN; advanced network services are required over existing campus, connector/regional, and national networks.

\subsection{Good End-to-End Performance}

To achieve good end-to-end performance, we need to diagnose (understand the limits of performance) and address (work with members and application communities to address those performance issues) [3]. Internet2 consists of campuses, corporations, regional networks, and the Internet2 backbone network. Our members care about connecting with other members, government labs and networks, and international partners. The Internet 2 community cares about making all of this work. Given that performance is excellent across backbone networks, and that performance is a problem end-to-end, it is clear that problems are concentrated towards the edge and in network transitions.

We envision readily available performance information that is easy to find, ubiquitous, reliable, valuable, actionable (analysis suggests course of action), and automated (applications act on data received). We assume a security system to authenticate and authorize users of the system as well as deter attacks. The goal of all this testing is to eliminate the mystery by increased network awareness. This allows us to set user expectations accurately, reduce diagnostic costs, and notice performance problems early, and address them efficiently. It also allows network engineers to see and act outside their "turf" as well as transforming application design to incorporate network intuition into application behavior. 
The strategy Internet2 is employing is building and empowering the community. This requires developing analysis and visualization tools [4], and encouraging performance data generation and sharing. For this to happen, we need clean APIs and protocols between each layer and widespread deployment of both the measurement infrastructure and a set of common performance measurement tools. Internet2 provides diagnostic information for its "U.S. backbone" portion of problem, and we are working closely with GÉANT2 to ensure that we are providing the type of data they are likely to require, as well as encouraging them to provide data needed by U.S. diagnosticians. Internet2 has created a few diagnostic tools (BWCTL, OWAMP, and Thrulay) and makes network data public.

In the coming year, the focus will be on making performance data more widely available via perfSONAR. Currently, Internet2 is contributing to the 'base' perfSONAR development effort in partnership with ESnet, European NRENs, and RNP (Brazil). Staff contributes to the development of standards for performance information sharing via the Open Grid Forum Network Measurement Working Group and are working to integrate the Internet2 diagnostic tools as examples of perfSONAR services to encourage development of tools in the community.

A network engineer or application easily can discover additional monitoring resources, authenticate locally, be authorized to use remote network resources to a limited extent, acquire performance monitoring data from remote sites via standard protocol, innovate where needed, and customize the analysis and visualization process.

For the radio astronomy community, the use of integrated network monitoring when conducting tests to determine the feasibility of e-VLBI (electronic Very Long Baseline Interferometry) helped enable identification of a hardware problem in time to correct it. Ongoing, automated monitoring allowed a view of network throughput variation over time and is now used to schedule short-term data transfers. The visualization of the data highlights route changes, network outages, and any throughput issues at end points. This integrated monitoring provides an overall view of network behavior at a glance for astronomers in the U.S., Japan, and Sweden; the goal is to extend this network of testing servers to all participating radio telescopes.

\subsection{Internet2 End-to-End Performance Initiative (E2Epi)}

E2Epi, which includes Internet2 staff, members, and federal and international partners building performance monitoring tools and frameworks, is supported via network revenues, partnerships, and grants from NSF and NLM. Work focuses on the development and widespread deployment of perfSONAR, an international consortium developing a performance middleware architecture and a set of protocol standards for inter-operability between measurement and monitoring systems. Overall, perfSONAR is a set of open source web services that can be mixed-and-matched and extended to create a performance monitoring framework. Design goals include being standards-based, modular, decentralized, open source, and extensible. This makes it applicable to multiple generations of network monitoring systems and encourages it to grow 'beyond our control,' while still allowing it to be customized for individual science applications. perfSONAR is a joint effort of ESnet, GÉANT2 JRA1, Internet2, and RNP.

For more information, either contact Eric Boyd <eboyd@internet2.edu> or see http://e2epi.internet2.edu/, http://www.perfsonar.net/, and http://nwmg.internet2.edu. 


\section{Network Measurement}

The original Abilene racks included measurement devices: a single PC coordinated early OWAMP and Surveyor measurements. The primary motivation was an understanding of how (and how well) the network operates. This was, largely, a NOC function but access to the measurements was available to other network operators to better understand the network. Over time, it became apparent that the datasets were valuable as a network research tool.

\subsection{The Abilene Observatory}

There are two components to the Observatory: 1) co-location (network research groups are able to co-locate equipment in the Abilene router nodes) and 2) measurement (data is collected by the NOC, the Ohio ITEC, and Internet2, and made available to the research community). During the Abilene upgrade in 2002; the network was expanded to two racks, one of which was dedicated to measurement. This provided the potential for the research community to co-locate equipment, which was immediately taken advantage of by several projects, including PlanetLab. For more information, see: http://abilene.internet2.edu/observatory/research-projects.html

\subsection{The Internet2 Network Observatory}

The Internet2 Network provides greater IP services than were available with Abilene and it requires a new type of Observatory to measure and monitor network activity. After seeking input from the community, Internet2 is supporting three types of services: 1) measurement, 2) co-location, and 3) experimental servers to support specific projects. The Internet2 Network Observatory will support both optical and router nodes. At this time, the Observatory collects data on one-way latency, jitter and loss; regularly-scheduled TCP and UDP throughput tests; SNMP; flow, with anonymized addresses; routing updates; router configuration; and dynamic updates (syslog, alarm generation, and polling via router proxy).

The Observatory uses Dell 1950 and Dell 2950 servers; they have Dual Core 3.0GHz Xeon processors, 2GB memory, Dual RAID 146GB disks, integrated 1GE copper interfaces, 10GE interfaces, and Hewlett-Packard 10GE switches. There are nine servers at router sites, with three servers at optical-only sites. Data is collected locally and stored in distributed databases; there are databases for usage, NetFlow, routing, latency, throughput, router, and syslog data. Some uses of existing datasets and tools include quality control, network diagnostics, network characterization, and network research. Modifications to the Observatory may be made in response to feedback from researchers.

\subsubsection{Quality Control (QC)}

Latency and throughput tests are required for any quality control effort. For Internet2 and its users, QC has been vital for some user communities (such as radio astronomers; see http://e2epi.internet2.edu/case-studies/VLBI/), network peerings, and IP backbone integrity. QC measurements for the latter have been vital to ensure problems are resolved in a timely manner. There are a full mesh of IPv4 and IPv6 tests on machines with 1GE interfaces (9000 MTU); the expected result is greater than 950Mbps TCP flows; if any path falls below 900Mbps for two successive testing intervals, this generates an alarm at the NOC. 
QC is also important for peerings; Internet2 and ESnet watch the latency across peering points; Internet2 and DREN also will conduct some throughput and latency testing. During the setup, engineers found interesting routing and MTU size issues that they are investigating.

\subsubsection{Network Diagnosis and Characterization}

This includes testing to end hosts and more generic testing. For the former, Internet2 uses NDT and NPAD servers; users can run a quick check from a host with a browser to eliminate (or confirm) last mile problems, such as buffer sizing, duplex mismatch, etc. NPAD finds switch limitations if the server is close enough. For the latter, tests generally look for configuration and loss. Flow data is collected with a flow-tools package; all data that is not used for security alerts and analysis [REN-ISAC] is anonymized by truncating the addresses. Reports from the anonymized data (and some engineering reports) are publicly available.

\subsubsection{Research Projects}

The four topics of primary interest to network researchers are: major consumption, flows, routes, and configuration. Thanks to NSF, a Network Research Facilities Grant made it possible for Internet2 to provide access to this information to network researchers for 1.5 years. The Internet2 Network Observatory (replacing the Abilene Observatory) contains inside racks set for initial deployment, including new research projects (NetFPGA, Phoebus). The software and links are easily changed and Internet2 easily could add or change hardware depending on costs. The new Observatory will provide researcher tools and new datasets. For more information, see http://www.internet2.edu/observatory/.

\section{References}

[1] Boote, J. W., Boyd, E. L., Durand, J., Hanemann, A., Kudarimoti, L., Lapacz, R., Simar, N., Trocha, S., Towards Multi-Domain Monitoring for the European Research Networks, in Selected Papers from the TERENA Networking Conference, TERENA, ISBN 90-77559-04-3, 2005; also published in PSNC's Computational Methods in Science and Technology series (Volume 11(2)).

[2] Hanemann, A., Boote, J. W., Boyd, E. L., Durand, J., Kudarimoti, L., Lapacz, R., Swany, D. M., Zurawski, J., Trocha, S., PerfSONAR: A Service Oriented Architecture for Multi-Domain Network Monitoring, in Proceedings of the Third International Conference on Service Oriented Computing, Springer Verlag, LNCS 3826, pp. 241-254, ACM Sigsoft and Sigweb, Amsterdam, The Netherlands, December, 2005.

[3] Hanemann, A., Liakopoulos, A., Molina, M., Swany, D. M., A Study on Network Performance Metrics and their Composition, in proceedings of TERENA Networking Conference 2006; also in special edition of Campus-Wide Information Systems (Volume 23 - 4 - 2006 - ISSN 1065-0741), Emerald Publishing Group Ltd.

[4] Hanemann, A., Jeliazkov, V., Kvittem, O., Marta, L., Metzger, J., Velimirovic, I., Complementary Visualization of perfSONAR Network Performance Measurements, in proceedings of International Conference on Internet Surveillance and Protection (ICISP), IARIA/IEEE, Cap Esterel, France, IARIA/IEEE, August, 2006. 